\title{
Robust Region Tracking Control for a Robot Manipulator
}

\author{
Xiangyun Li, Qi Lu, Member, IEEE, Zhaoyang Chen, \\ Qinlin Yang, and Kang Li, Member, IEEE
}

\begin{abstract}
In this work, the uncertainty and disturbance estimator (UDE)-based robust region reaching controller for a robot manipulator is developed to achieve the moving target region trajectory tracking and complaint human robot interaction inside the target region. The regional feedback error is derived from the potential function to drive the robot manipulator end effector converging into the target region. Under the back-stepping control framework, the UDE is fused into the region tracking control law to estimate and compensate the model uncertainty and external disturbance. Both simulation and experimental studies are carried out with a universal robots (UR) 10 manipulator to demonstrate the effectiveness of the proposed method for moving target trajectory tracking, model uncertainty and external disturbance rejection, and compliant human robot interaction within the target region.
\end{abstract}

Note to Practitioners - This work is motivated by the motion control problem of the robot manipulator with human robot interaction capability for carrying out the nursing tasks during COVID-19 pandemic. The proposed control method considers the region tracking instead of the set-point tracking to allow for compliant human robot interaction within the target region. To deal with the model uncertainty and external disturbance which are inevitable for robot manipulator controller implementation in real applications, the uncertainty and disturbance estimator (UDE) is integrated into region tracking control framework. Due to the limitation of the experimental platform, where the torque cannot be directly controlled, which are quite common for industrial robot manipulators, the proposed approach is indirectly implemented by sending the joint angle commands. The experimental results validated that the proposed controller can be implemented on real robot manipulator platform with sufficient robustness and compliant human robot interaction capability. In the future research, the tele-operation control modules will be incorporated.

Index Terms-region tracking control, uncertainty and disturbance estimator, robot manipulator, human robot interaction.

\section{INTRODUCTION}

$\mathbf{T}$ HE outbreak of the novel corona-virus disease 2019 (COVID-19) which features high contagiousness and infection fatality rate has now become a once-in-a-century

Xiangyun $\mathrm{Li}$, Zhaoyang Chen and Kang $\mathrm{Li}$ are with the West China Biomedical Big Data Center, West China Hospital, Sichuan University, Chengdu, Sichuan, 610207 China (e-mail: xiangyun.app@gmail.com; czy98@hotmail.com; likang@wchscu.cn).

Qi Lu (corresponding author) is with the Department of Mechanical Engineering, Sichuan University-Pittsburgh Institute, Sichuan University, Chengdu, Sichuan, 610207 China (e-mail: qi.lu@ @scu.edu.cn).

Qinlin Yang is with the Department of Automation, College of Electric Engineering, Sichuan University, Chengdu, Sichuan, 610207 China (e-mail: qinlin.yang@hotmail.com). pandemic [1], [2]. At the time of writing, this pandemic has affected 219 countries and territories around the world with more than 99 million confirmed cases [3]. Healthcare workers, like nurses and doctors are in direct contact with the patients who carries the highly infectious coronavirus, putting themselves and possibly their families at high risk of infection [4]. With the benefits of using the robotic systems for combating the COVID-19, including intrinsic immunity to the virus, intensive and extra long hour service capability, process automation and multi-functional testing, the personal exposure to the patients and the labor intensity of the healthcare workers are largely reduced [5], [6]. Therefore, various researchers have investigated the use of robot manipulator for carrying out the healthcare tasks, like throat swabbing [7], nasal swapping [8] and ultrasound testing [9].

Robot manipulator control, especially for high degreesof-freedom (DOFs) system, is a challenging task due to the nonlinear system dynamics, kinematic uncertainties from interchangeable end-effectors with different dimensions and dynamic uncertainties from objects with unknown mass [10][12]. The static and kinematic frictions also create nonlinear disturbance causing the motor torque loss up to $30 \%$ [13]. The dynamic behavior of the manipulator is highly associated with the control system design. The adaptive and robust controllers are developed to maintain performance in terms of stability, tracking error, or other specifications, despite parametric uncertainties, external disturbances and unmodeled dynamics presented in the real manipulator system. The investigation of the adaptive control methods for robot manipulator has a long history. The employment of adaptive control provides the system with the capability of real time parametric uncertainty estimations; thus, enabling the robot manipulators to perform tasks in the unknown environment [14]. In [15], Cheah et al. proposes an adaptive feedback control law for robot manipulator setpoint control in task space to deal with the kinematic uncertainty. The developed controller can handle the uncertain Jacobian matrix and uncertain gravitational force with simulation validation. Then the proposed method is extended to handle the kinematic and dynamic uncertainties simultaneously in [16]. Besides the transpose Jacobian approach, the inverse Jacobian regulator has been invested in [17]. Considering the physical constraints of the actuators, the torque amplitude limited adaptive control is proposed in [10]. To deal with the implementation issue with the industrial robot and ensure the transient performance of the system, the adaptive controllers which enjoy the separation properties are proposed in [18]. The 
dynamic behavior of the inner loop controller, which comes with the robot manipulator, is incorporated into the outer loop controller design, which is specified by the user, to grantee the stability and control performance of the overall closedloop system.

The development of the adaptive controller usually relies on the assumption of linearly parameterized system [19]. To handle the uncertain nonlinearities and external disturbance, the robust control method is usually adopted. The novel robust model predictive control that unifies the planning and control layers is proposed in [20], where the neural networks is utilized to approximate the piece-wise constant model predictive control input to reduce the computation time. The sliding mode control (SMC) is a nonlinear robust control method that alters the dynamics of a system by the application of a discontinuous control signal which forces the system to slide along the sliding surface. By adding adaptive term to a robust control algorithm, i.e. sliding mode controller, the superior performance of the proposed controller is demonstrated with an industrial robot manipulator in [19] with comparison to the controller without adaptive term. In [21], the integral suboptimal second order sliding mode control algorithm is developed to reduce the reaching phase and enhance the robustness; experimental results demonstrated the better robustness of the proposed method compared to conventional PD control. The time-delay control (TDC) utilizes the time-delayed information at the previous sampling constant to estimate the external disturbance. To enhance the performance of the TDC, Jin et al. adopts a nonlinear sliding mode-based time delay estimation error correction term, making the controller has a inclusive structure [13]. The TDC with the adaptive gain dynamics is investigated in [22] to handle the significant payload changes for robot manipulator. Instead of using a delay term, the uncertainty and disturbance estimator (UDE)-based control [23] employs a filter to estimate the uncertainty and disturbance, thus bringing the benefits of no delay in the system and no oscillations in the control signal. The UDE-based control has been successfully applied to the motion control of various mechanical systems, including quadrotor [24] and piezoelectric stage [25].

Before the robot manipulator can be practically deployed, the physical human-robot interaction capability needs to guaranteed [5]. Usually, the end-effector path in the task space is specified as a series of set-points [10], [22], [26]-[28]. For applications which involves human robot interaction or cooperation, it is more desirable to set the target for the robot end-effector as a region instead of a point in Cartesian space. The robot manipulator will exhibit compliant behavior inside the target region to ensure the safe and smooth physical human-robot interaction. For example, a recently developed assistive feeding robot can help the handicapped patient to complete the eating task by delivering the soup using a spoon to a target area near the patient's mouth. Then the patient will guide the motion of the end-effector with the palm to accomplish final step [29]. Another example would be the robot assisted rehabilitation, which involves the passive and active phases, the robot would assist the movement of the patient during the passive rehabilitation phase, helping the patient reaching the active rehabilitation region. In the active rehabilitation region, the compliant behavior is desired as the patient takes initiative to accomplish the rest of the work [30], [31]. The concept of region reaching control for robot manipulator is originally proposed in [32]. Compared to the conventional set-point control, the region reaching control can provide more flexibility and it has the advantages of faster convergence with less control effort. Therefore, it has been widely investigated in robot manipulator adaptive control [33], [34], flexible-joint manipulator control [29], human-guided robotic co-manipulation [35], and optical tweezers control [36].

Based on the above discussions, an UDE-based robust region tracking controller for the robot manipulator motion control is developed in this work by utilizing the back-stepping control framework and Lyapunov control design method. The compliant behavior of the robot manipulator for physical human-robot interaction is achieved within the target region. The model uncertainty and external disturbance is estimated and compensated by the UDE. The main contributions of this article are listed as follows:

(1) The regional feedback error is adopted to achieve the moving target region trajectory tracking with fast convergence and less control effort. Furthermore, the safe and smooth physical human-robot interaction is achieved within the target region.

(2) Compared with the exiting works for adaptive region reaching robot manipulator control [33]-[35], the proposed robust region tracking control method incorporate the UDE to estimate and compensate the model uncertainty and external disturbance simultaneously.

(3) Both simulation and experimental validations are carried out to demonstrate the effectiveness of the proposed methods, including trajectory tracking, uncertainty and disturbance rejection and human-robot interaction.

The rest of this article is organized as follows. Section II presents the preliminaries about the robot manipulator dynamics and the problem formulation. Section III discusses the developments of the UDE-based robust region tracking controller design. The effectiveness of the proposed approach is demonstrated through simulations and experiments in Section IV. Section V concludes this article.

\section{System DyNAMiCS AND PROBLEM Formulation}

\section{A. System Dynamics}

Let $\mathbf{x} \in \mathbb{R}^{3}$ represent the position vector of the end effector in task space

$$
\mathbf{x}=h(\mathbf{q})
$$

where $\mathbf{q} \in \mathbb{R}^{n}$ is a vector of generalized joint coordinates for $n$ DOFs manipulator, $h(\cdot)$ is the transformation matrix which maps the joint angle $\mathbf{q}$ in the joint space to end-effector position $\mathbf{x}$ in the task space. Taking the time derivative of (1), the kinematic relationship between the velocity vector of the end effector $\dot{\mathbf{x}}$ and the joint velocity velocity vector $\dot{\mathbf{q}}$ can be described as

$$
\dot{\mathbf{x}}=\mathbf{J}(\mathbf{q}) \dot{\mathbf{q}}
$$


where $\mathbf{J}(\mathbf{q})$ is the Jacobian matrix. The dynamics the robot manipulator in the joint space can be described as

$$
\mathbf{M}(\mathbf{q}) \ddot{\mathbf{q}}+\mathbf{C}(\mathbf{q}, \dot{\mathbf{q}}) \dot{\mathbf{q}}+\mathbf{g}(\mathbf{q})=\boldsymbol{\tau}+\boldsymbol{\tau}_{d}
$$

where $\mathbf{M}(\mathbf{q}) \in \mathbb{R}^{n \times n}$ is the inertia matrix, $\mathbf{g}(\mathbf{q}) \in \mathbb{R}^{n}$ denotes a gravitational torque vector, $\mathbf{C}(\mathbf{q}, \dot{\mathbf{q}}) \dot{\mathbf{q}} \in \mathbb{R}^{n}$ is the Coriolis and centrifugal torque, $\boldsymbol{\tau}$ denotes the control torques and $\tau_{d}$ is the bounded disturbance torque term, which includes the effects of friction, external disturbance and model uncertainty. From the forward kinematics (2), $\dot{\mathbf{q}}$ and $\ddot{\mathbf{q}}$ can be derived as

$$
\begin{aligned}
\dot{\mathbf{q}} & =\mathbf{J}^{-1}(\mathbf{q}) \dot{\mathbf{x}} \\
\ddot{\mathbf{q}} & =\dot{\mathbf{J}}^{-1}(\mathbf{q}) \dot{\mathbf{x}}+\dot{\mathbf{J}}^{-1}(\mathbf{q}) \ddot{\mathbf{x}}
\end{aligned}
$$

The task space dynamics can be derived by combining the joint space dynamics (3) and (4) [37]

$$
\mathbf{M}_{\mathbf{x}}(\mathbf{x}) \ddot{\mathbf{x}}+\mathbf{C}_{\mathbf{x}}(\mathbf{x}, \dot{\mathbf{x}}) \dot{\mathbf{x}}+\mathbf{g}(\mathbf{x})=\mathbf{F}+\mathbf{F}_{d}
$$

where

$$
\begin{aligned}
\mathbf{M}_{\mathbf{x}}(\mathbf{x}) & =\mathbf{J}^{-\mathbf{T}}(\mathbf{q}) \mathbf{M}(\mathbf{q}) \mathbf{J}^{-1}(\mathbf{q}) \\
\mathbf{C}_{\mathbf{x}}(\mathbf{x}, \dot{\mathbf{x}}) & =\mathbf{J}^{-\mathbf{T}}\left(\mathbf{C}(\mathbf{q}, \dot{\mathbf{q}})-\mathbf{M}(\mathbf{q}) \mathbf{J}^{-1}(\mathbf{q}) \dot{\mathbf{J}}(\mathbf{q})\right) \mathbf{J}^{-1}(\mathbf{q}) \\
\mathbf{g}(\mathbf{x}) & =\mathbf{J}^{-\boldsymbol{\top}} \mathbf{g}(\mathbf{q}) \\
\mathbf{F} & =\mathbf{J}^{-\boldsymbol{\top}} \boldsymbol{\tau} \\
\mathbf{F}_{d} & =\mathbf{J}^{-\boldsymbol{T}} \boldsymbol{\tau}_{d}
\end{aligned}
$$

and the following properties holds [18]:

Property 1: The inertia matrix $\mathbf{M}_{\mathbf{x}}(\mathbf{x})$ is symmetric and positive definite.

Property 2: The matrix $\dot{\mathbf{M}}_{\mathbf{x}}(\mathbf{x})-2 \mathbf{C}_{\mathbf{x}}(\mathbf{x}, \dot{\mathbf{x}})$ is a skewsymmetric matrix.

In this work, the control tasks are all specified in the robot manipulator task space. Therefore, it will be more convenient to design the controller in the task space directly, which necessitates the coordinate transformation from the joint space to the task space.

\section{B. Problem Formulation}

The objective of this paper is to design the UDE-based robust region tracking control algorithm for the robot manipulator such that the position of the end effector will be regulated to and track a moving target region, even in the presence of nonlinearity, model uncertainty and external disturbance. The shape of the moving target region $\Omega$ is chosen as a ball with the center defined as the point $\mathbf{x}_{o} \in \mathbb{R}^{3}$ and the radius defined as $r_{o}$. It is assumed that the trajectory of the center point $\mathbf{x}_{O}$ is second order differentiable.

\section{Controller Design}

\section{A. Potential Energy Functions}

The target region can be expressed as a compact set in three dimensional space as [38]

$$
\Omega=\left\{\Delta \mathbf{x} \mid f(\Delta \mathbf{x})=\|\Delta \mathbf{x}\|^{2}-r_{o}^{2} \leq 0\right\}
$$

where $\Delta \mathbf{x}=\mathbf{x}-\mathbf{x}_{o}, \mathbf{x}_{o}$ is the center of the target region, $f(\Delta \mathbf{x}): \mathbb{R}^{3} \rightarrow \mathbb{R}$ is the objective function. The target function is chosen to be continuous and differentiable with respect to $\Delta \mathbf{x}$ such that the boundedness of $f(\Delta \mathbf{x})$ assures the boundedness of the first and second partial derivatives, which are $\frac{\partial f(\cdot)}{\partial \Delta \mathbf{x}}$ and $\frac{\partial^{2} f(\cdot)}{\partial^{2} \Delta \mathbf{x}}$. the potential energy function for the manipulator is designed as

$$
P(\Delta \mathbf{x})=\frac{c}{2} \max (0, f(\Delta \mathbf{x}))^{2}
$$

where $c$ is a positive constant. The target potential energy function $P(\Delta \mathbf{x})$ is continuous differentiable with respect to $\Delta \mathrm{x}$ and monotonically increases with $\|\Delta \mathrm{x}\|$. Partially differentiating the potential function $P(\Delta \mathbf{x})$ with respect to $\Delta \mathrm{x}$ leads to

$$
\begin{aligned}
\frac{\partial P(\Delta \mathbf{x})}{\partial \Delta \mathbf{x}} & =c \max (0, f(\Delta \mathbf{x})) \frac{\partial f(\Delta \mathbf{x})}{\partial \Delta \mathbf{x}} \\
& =2 c \max (0, f(\Delta \mathbf{x})) \Delta \mathbf{x}
\end{aligned}
$$

where $\frac{\partial P(\Delta \mathbf{x})}{\partial \Delta \mathbf{x}}$ is a column vector. It can be seen that from (8) that when the manipulator end effector is outside the target region $\Omega, \frac{\partial P(\Delta \mathbf{x})}{\partial \Delta \mathbf{x}}$ is activated to drive the manipulator end effector towards the target region and when the manipulator end effector is inside the target region $\Omega, \frac{\partial P(\Delta \mathbf{x})}{\partial \Delta \mathbf{x}}$ remains as zero.

\section{B. Region Tracking Controller Design}

In this subsection, the region tracking position controllers for the robot manipulator will be designed with the backstepping technique. Denote the error coordinates

$$
\begin{aligned}
& \mathbf{z}_{1}=\Delta \mathbf{x}=\mathbf{x}-\mathbf{x}_{o} \\
& \mathbf{z}_{2}=\dot{\mathbf{x}}-\dot{\mathbf{x}}_{o}-\boldsymbol{\nu}
\end{aligned}
$$

where $\boldsymbol{\nu}$ are the virtual control vectors. Taking the time derivative of (10) leads to

$$
\begin{aligned}
\dot{\mathbf{z}}_{1} & =\dot{\mathbf{x}}-\dot{\mathbf{x}}_{o}=\mathbf{z}_{2}+\boldsymbol{\nu} \\
\dot{\mathbf{z}}_{2} & =\ddot{\mathbf{x}}-\ddot{\mathbf{x}}_{o}-\dot{\boldsymbol{\nu}} \\
& =\mathbf{M}_{\mathbf{x}}^{-1}(\mathbf{x})\left[\mathbf{F}+\mathbf{F}_{d}-\mathbf{C}_{\mathbf{x}}(\mathbf{x}, \dot{\mathbf{x}}) \dot{\mathbf{x}}-\mathbf{g}(\mathbf{x})\right]-\ddot{\mathbf{x}}_{o}-\dot{\nu}
\end{aligned}
$$

Consider the following Lyapunov function candidate

$$
V=\frac{1}{2} \mathbf{z}_{2}^{\top} \mathbf{z}_{2}+P(\Delta \mathbf{x})
$$

The time derivative of (12) is

$$
\dot{V}=\mathbf{z}_{2}^{\top} \dot{\mathbf{z}}_{2}+\left(\frac{\partial P(\Delta \mathbf{x})}{\partial \Delta \mathbf{x}}\right)^{\top} \Delta \dot{\mathbf{x}}
$$

Substituting (11) into (13) leads to

$$
\begin{aligned}
\dot{V} & =\mathbf{z}_{2}^{\top}\left\{\mathbf{M}_{\mathbf{x}}^{-1}(\mathbf{x})\left[\mathbf{F}+\mathbf{F}_{d}-\mathbf{C}_{\mathbf{x}}(\mathbf{x}, \dot{\mathbf{x}}) \dot{\mathbf{x}}-\mathbf{g}(\mathbf{x})\right]-\ddot{\mathbf{x}}_{o}-\dot{\boldsymbol{\nu}}\right\} \\
& +\Delta \dot{\mathbf{x}}^{\top} \frac{\partial P(\Delta \mathbf{x})}{\partial \Delta \mathbf{x}} \\
& =\mathbf{z}_{2}^{\top}\left\{\mathbf{M}_{\mathbf{x}}^{-1}(\mathbf{x})\left[\mathbf{F}+\mathbf{F}_{d}-\mathbf{C}_{\mathbf{x}}(\mathbf{x}, \dot{\mathbf{x}}) \dot{\mathbf{x}}-\mathbf{g}(\mathbf{x})\right]-\ddot{\mathbf{x}}_{o}-\dot{\boldsymbol{\nu}}\right\} \\
& +\left(\mathbf{z}_{2}+\boldsymbol{\nu}\right)^{\top} \frac{\partial P(\Delta \mathbf{x})}{\partial \Delta \mathbf{x}}
\end{aligned}
$$

Consider the virtual controller in the form of

$$
\boldsymbol{\nu}=-\mathbf{K}^{\nu} \frac{\partial P(\Delta \mathbf{x})}{\partial \Delta \mathbf{x}}
$$


where $\mathbf{K}^{\nu} \in \mathbb{R}^{3 \times 3}$ are positive constant matrices. Combining (14) and (15) lead to

$$
\begin{aligned}
\dot{V} & =\mathbf{z}_{2}^{\top}\left\{\mathbf{M}_{\mathbf{x}}^{-1}(\mathbf{x})\left[\mathbf{F}+\mathbf{F}_{d}-\mathbf{C}_{\mathbf{x}}(\mathbf{x}, \dot{\mathbf{x}}) \dot{\mathbf{x}}-\mathbf{g}(\mathbf{x})\right]\right. \\
& \left.-\ddot{\mathbf{x}}_{o}-\dot{\boldsymbol{\nu}}+\frac{\partial P(\Delta \mathbf{x})}{\partial \Delta \mathbf{x}}\right\}-\left(\frac{\partial P(\Delta \mathbf{x})}{\partial \Delta \mathbf{x}}\right)^{\top} \mathbf{K}^{\nu}\left(\frac{\partial P(\Delta \mathbf{x})}{\partial \Delta \mathbf{x}}\right)
\end{aligned}
$$

To ensure the derivative of the Lyapunov function is negative definite, the control action term is designed as

$$
\begin{aligned}
\mathbf{F} & =\mathbf{C}_{\mathbf{x}}(\mathbf{x}, \dot{\mathbf{x}}) \dot{\mathbf{x}}+\mathbf{g}(\mathbf{x})-\hat{\mathbf{F}}_{d}+\mathbf{M}_{\mathbf{x}}(\mathbf{x})\left[\ddot{\mathbf{x}}_{o}+\dot{\nu}\right. \\
& \left.-\frac{\partial P(\Delta \mathbf{x})}{\partial \Delta \mathbf{x}}-\mathbf{K}^{P} \mathbf{z}_{2}\right]
\end{aligned}
$$

where $\mathbf{K}^{P} \in \mathbb{R}^{3 \times 3}$ are positive constant matrices and $\hat{\mathbf{F}}_{d}$ is the estimation of the disturbance term $\mathbf{F}_{d}$. If the disturbance term is measurable, then results in

$$
\dot{V}=-\mathbf{z}_{2}^{\top} \mathbf{K}^{P} \mathbf{z}_{2}-\left(\frac{\partial P(\Delta \mathbf{x})}{\partial \Delta \mathbf{x}}\right)^{\top} \mathbf{K}^{\nu}\left(\frac{\partial P(\Delta \mathbf{x})}{\partial \Delta \mathbf{x}}\right)
$$

However, the disturbance term is unpredictable and unmeasurable. By adopting stable strictly proper filters with unity steady-state gains, the UDE technique provided in [23] is used to construct the disturbance estimation as

$$
\begin{aligned}
\hat{\mathbf{F}}_{d}= & \mathcal{L}^{-1}\{\mathbf{G}(s)\} *\left[\mathbf{M}_{\mathbf{x}}(\mathbf{x})\left(\dot{\mathbf{z}}_{2}+\ddot{\mathbf{x}}_{o}+\dot{\boldsymbol{\nu}}\right)\right. \\
& \left.-\mathbf{F}+\mathbf{C}_{\mathbf{x}}(\mathbf{x}, \dot{\mathbf{x}}) \dot{\mathbf{x}}+\mathbf{g}(\mathbf{x})\right]
\end{aligned}
$$

where $\mathcal{L}^{-1}$ is the inverse Laplace operator, $*$ is the convolution operator, $\mathbf{G}(s)$ is a filter matrix. Substituting (17) back into (16) and solving for the control inputs $\mathbf{F}$ results in

$$
\begin{aligned}
\mathbf{F} & =\mathbf{C}_{\mathbf{x}}(\mathbf{x}, \dot{\mathbf{x}}) \dot{\mathbf{x}}+\mathbf{g}(\mathbf{x})+\mathbf{M}_{\mathbf{x}}(\mathbf{x})\left(\ddot{\mathbf{x}}_{o}+\dot{\boldsymbol{\nu}}\right) \\
& -\mathcal{L}^{-1}\left\{\left(\mathbb{I}_{3}-\mathbf{G}(s)\right)^{-1}\right\} * \mathbf{M}_{\mathbf{x}}(\mathbf{x})\left[\frac{\partial P(\Delta \mathbf{x})}{\partial \Delta \mathbf{x}}+\mathbf{K}^{P} \mathbf{z}_{2}\right] \\
& -\mathcal{L}^{-1}\left\{\left(\mathbb{I}_{3}-\mathbf{G}(s)\right)^{-1} \mathbf{G}(s) s\right\} * \mathbf{M}_{\mathbf{x}}(\mathbf{x}) \mathbf{z}_{2}
\end{aligned}
$$

where $\mathbb{I}_{n} \in \mathbb{R}^{n \times n}$ is the $n$-dimensional identity matrix.

\section{Results AND Discussion}

In this section, the numerical simulations and experimental studies are carried out to validate the effectiveness of the developed UDE-based robust region tracking controllers. The universal robots (UR) 10 robot manipulator which has six revolute joints, a load capacity of $10 \mathrm{~kg}$ and a operation range of $1300 \mathrm{~mm}$ is used as the validation platform, as shown in Fig. 1 (a). The symbolic representation of the UR 10 robot manipulator is shown in Fig. 1 (b) along with the Denavit and Hartenberg (DH) parameters shown in Table. I. Only the motions of the lower three joints are considered, the positions of its upper three joints are set as fixed value in both simulation and experiments.

In the simulation and experimental implementation, the low pass filter matrix $\mathbf{G}(s)$ and the controller parameter matrices $\mathbf{K}^{\nu}, \mathbf{K}^{P}$ in (18) are designed as diagonal matrices in the forms of $\mathbf{G}(s)=\operatorname{diag}\left(\frac{1}{T_{1} s+1}, \frac{1}{T_{2} s+1}, \frac{1}{T_{3} s+1}\right), \mathbf{K}^{\nu}=$ $\operatorname{diag}\left(k_{1}^{\nu}, k_{2}^{\nu}, k_{3}^{\nu}\right)$ and $\mathbf{K}^{P}=\operatorname{diag}\left(k_{1}^{P}, k_{2}^{P}, k_{3}^{P}\right)$, respectively, where $\operatorname{diag}(\cdot)$ is the diagonal matrix operator. The positive constant $c$ is set as $c=1$. The controller parameter choices for UR10 are listed in Table. II.

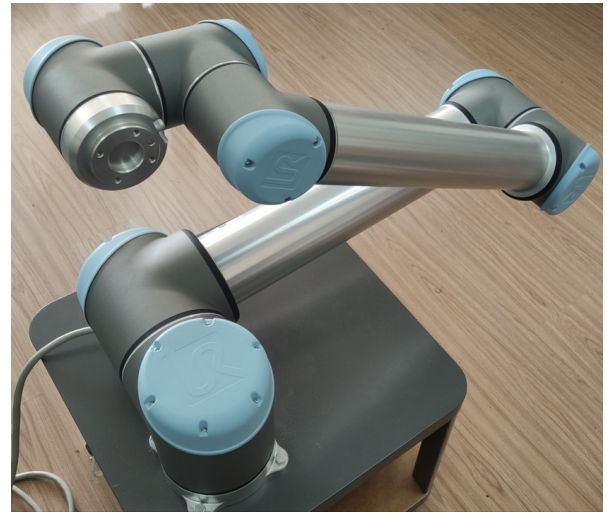

(a)

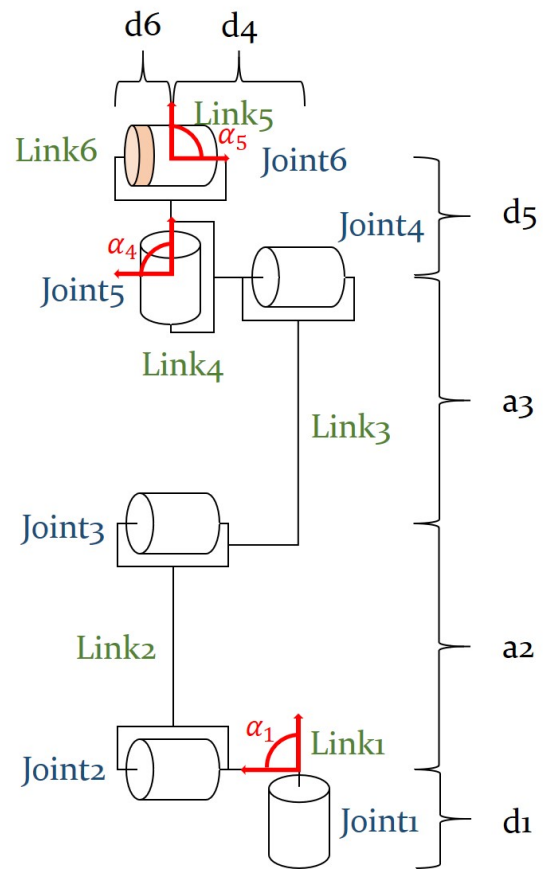

(b)

Fig. 1. Experimental platform: UR 10 robot manipulator and its symbolic representation. (a) UR 10 robot manipulator, (b) symbolic representation of UR 10 .

TABLE I

THE DH PARAMETER OF THE UR 10 ROBOT

\begin{tabular}{|c|c|c|c|}
\hline Parameter & $a(\mathrm{~m})$ & $d(\mathrm{~m})$ & $\alpha(\mathrm{rad})$ \\
\hline \hline Joint 1 & 0 & 0.118 & $\frac{\pi}{2}$ \\
\hline Joint 2 & -0.6127 & 0 & 0 \\
\hline Joint 3 & -0.5716 & 0 & 0 \\
\hline Joint 4 & 0 & 0.1639 & $\frac{\pi}{2}$ \\
\hline Joint 5 & 0 & 0.1157 & $-\frac{\pi}{2}$ \\
\hline Joint 6 & 0 & 0.0922 & 0 \\
\hline
\end{tabular}

\section{A. Simulation Results}

The effectiveness of the developed UDE-based robust region tracking controller is firstly validated with numerical simulations using Matlab 2020a. The fixed-step ODE4 (RungeKutta) solver with the time step of $0.005 \mathrm{~s}$ is utilized. The simulation model dynamics is built based on the UR10 robot manipulator and the total masses of the first three links are listed in Table III. The initial positions of the lower three joints 

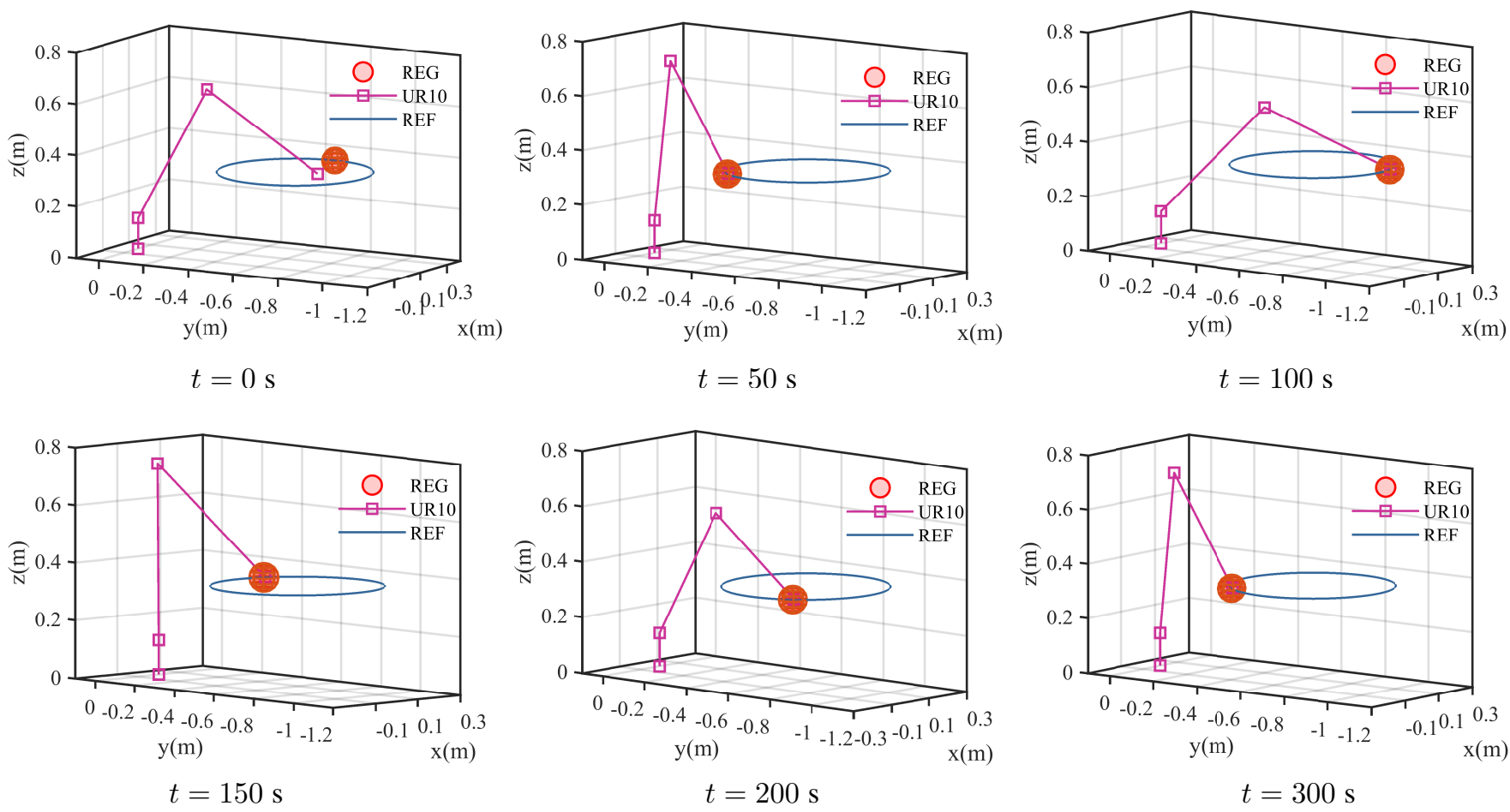

Fig. 2. Simulation case I: moving region trajectory tracking: 3D view of the end effector trajectory. REG: target region, UR10: robot manipulator end effector trajectory, REF: reference trajectory.

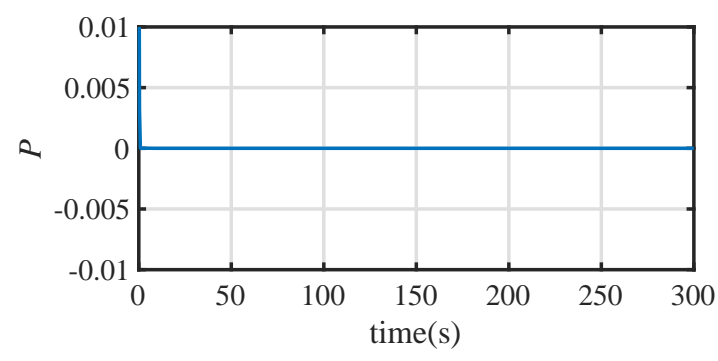

Fig. 3. Simulation case I: potential energy function.

TABLE II

Controller Parameter Selection for UR10

\begin{tabular}{|c|c|c|c|}
\hline$i$ th joint & $T_{i}$ & $k_{i}^{\nu}$ & $k_{i}^{P}$ \\
\hline \hline 1 & $1 / 30$ & 1 & 1 \\
\hline 2 & $1 / 30$ & 1 & 1 \\
\hline 3 & $1 / 30$ & 1 & 1 \\
\hline
\end{tabular}

set as $\left[\begin{array}{lll}\pi / 2 & -\pi / 3 & \pi / 2\end{array}\right]^{\top}$ and the upper three joints are kept as fixed value of $\left[\begin{array}{lll}0 & -\pi / 2 & 0\end{array}\right]^{\top} \mathrm{rad}$.

TABLE III

UR10 PHYSICAL PARAMETERS USED IN SIMULATION

\begin{tabular}{|c|c|c|c|}
\hline Parameters & $m_{1}$ & $m_{2}$ & $m_{3}$ \\
\hline \hline Values & $7.10 \mathrm{~kg}$ & $12.70 \mathrm{~kg}$ & $4.27 \mathrm{~kg}$ \\
\hline
\end{tabular}
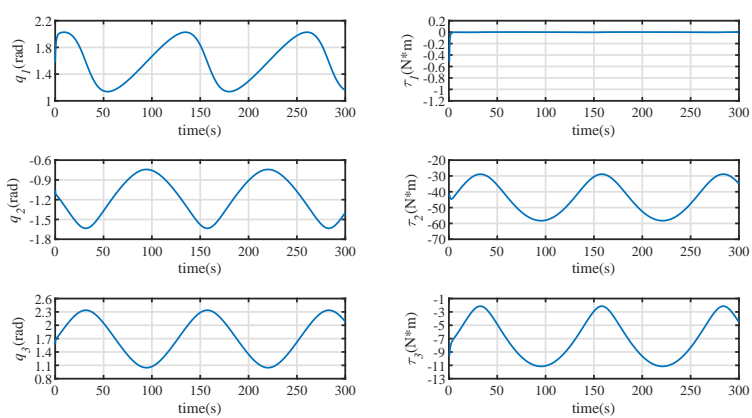

(a)

(b)

Fig. 4. Simulation case I: joint angles and control inputs. (a) joint angles (b) control inputs.

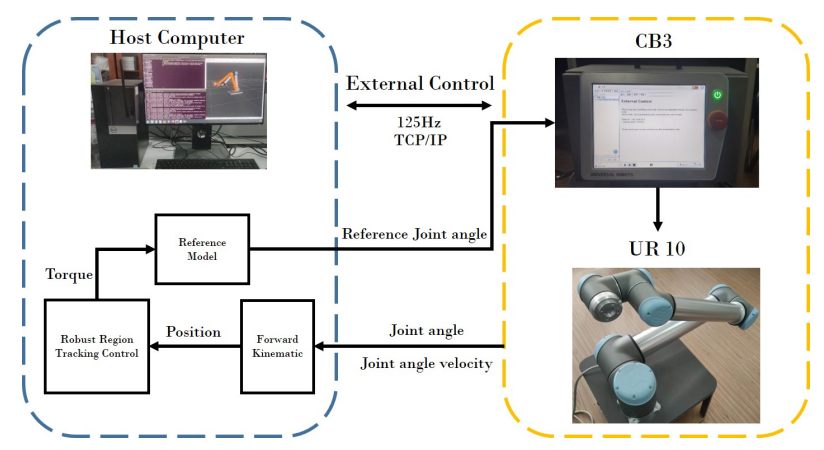

Fig. 8. Experimental setup. 

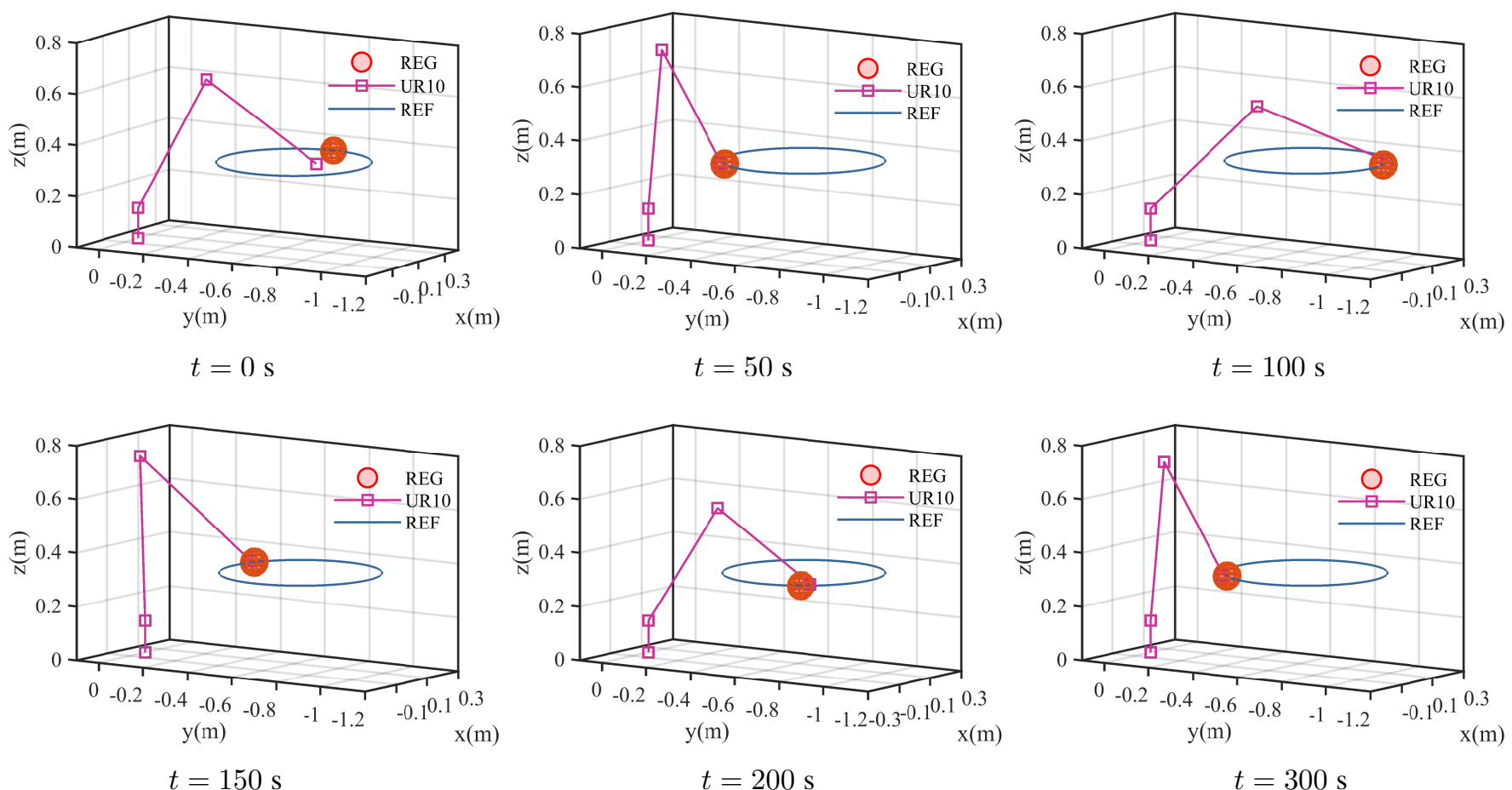

Fig. 5. Simulation case II: uncertainty and disturbance rejection: 3D view of the end effector trajectory. REG: target region, UR10: robot manipulator end effector trajectory, REF: reference trajectory.

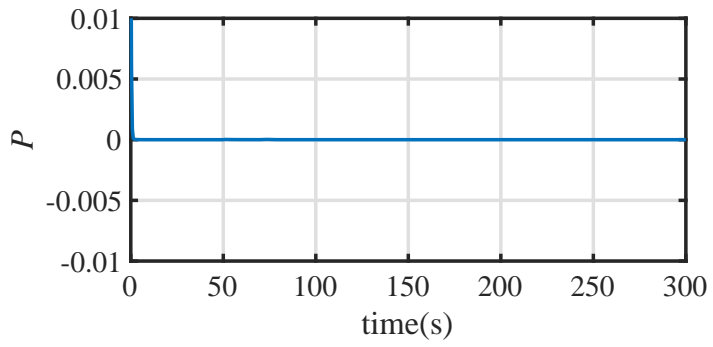

Fig. 6. Simulation case II: potential energy function.

Simulation Case I: Moving Region Trajectory Tracking: The control objective of this case is to steer the end-effector of the UR10 robot to track the moving target region, which is defined as a ball with a radius of $r=0.05 \mathrm{~m}$. The center of the target region $x_{o}$ moves along the desired trajectory $\mathbf{x}_{o}=[0.3 \cos (0.05 t),-0.8+0.3 \sin (0.05 t), 0.2]^{\top}$. Fig. 2 shows the snapshots of the end effector trajectory over the time period of $300 \mathrm{~s}$ in 3D view. The potential energy function is shown in Fig. 3. It can be observed that the robot end effector can quickly converge into the target region and track the moving region trajectory. The time evolutions of the joints angles and control inputs are shown in Fig. 4. This simulation results have successfully demonstrated the effectiveness of the UDE-based robust region tracking control algorithm for accomplishing the moving trajectory tracking task.

Simulation Case II: Uncertainty and Disturbance Rejection: When accomplishing different tasks, the robot manipulator
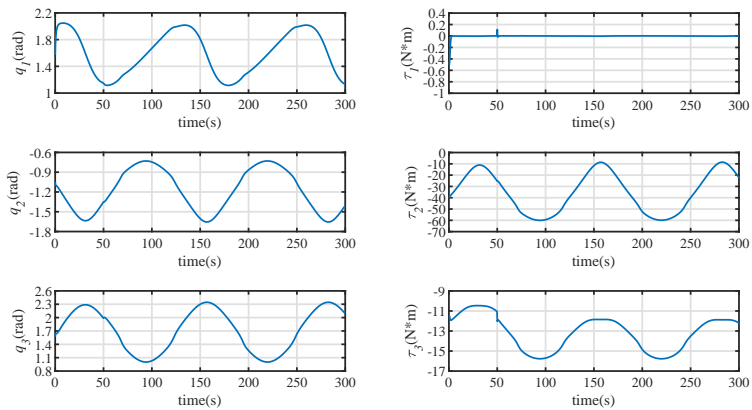

(a)

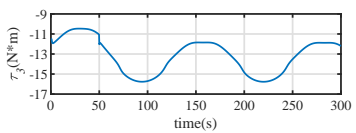

(b)

Fig. 7. Simulation case II: joint angles and control inputs. (a) joint angles (b) control inputs.

may need to pick up different tools with various masses which results in model uncertainties and the frictions also causes external disturbance. To test the robustness of the proposed control strategies, the second simulation case is carried out with the control objective as driving the robot manipulator end effector to track the moving region trajectory in presence of model uncertainty and external disturbance. The trajectory considered is the same as the simulation case I without modifying the controller parameters. The model uncertainty is considered as an additional tool with the mass of $0.5 \mathrm{~kg}$ added to the robot manipulator end effector at the beginning of the simulation. The mass parameter of the tool is unkown to the controller. Then the disturbance torques with the magnitude of $1 \mathrm{~N} \cdot \mathrm{m}$ are added to the three joints at 50 

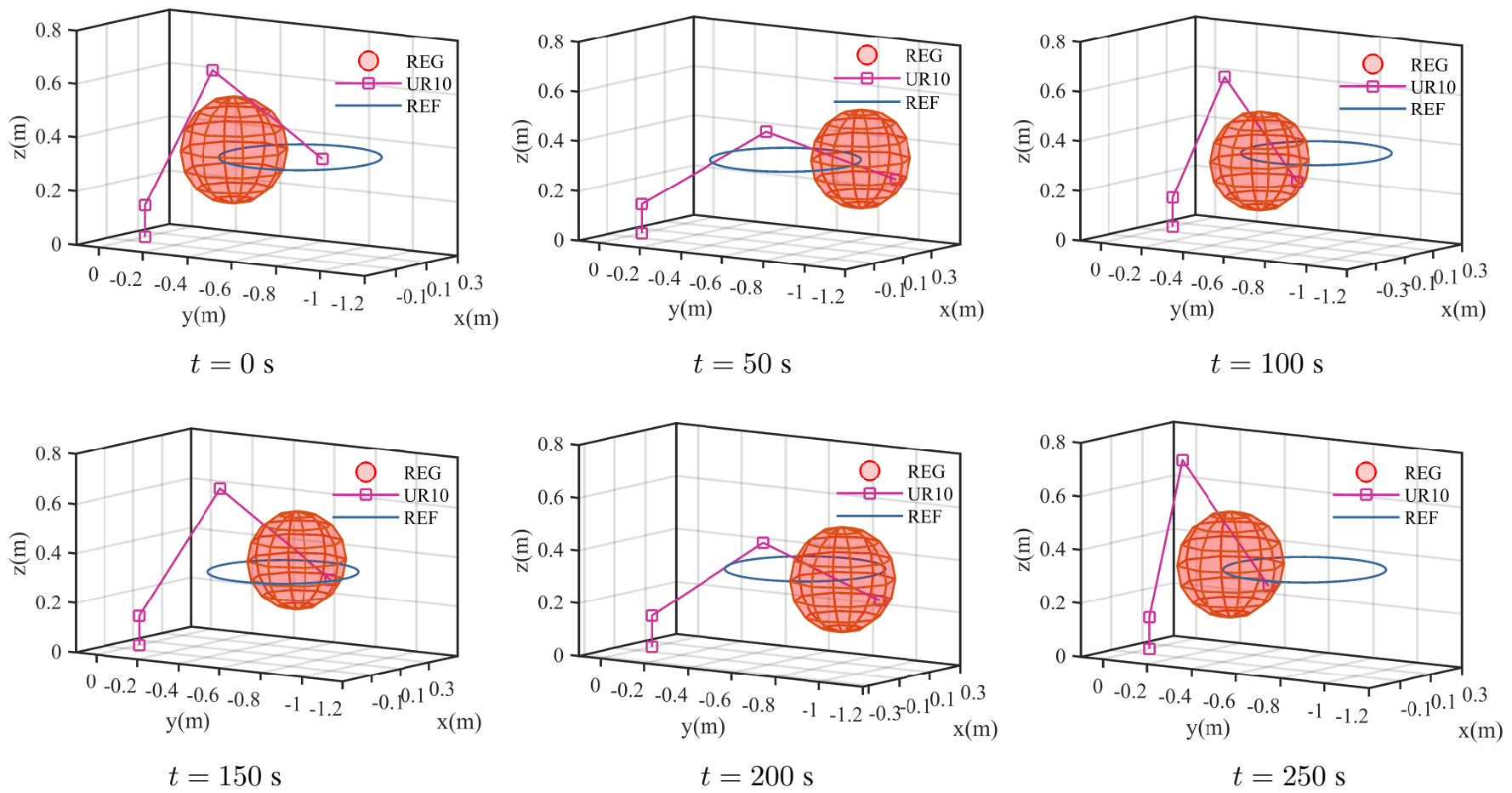

$t=250 \mathrm{~s}$

Fig. 9. Experimental case I: moving region trajectory tracking: 3D view of the end effector trajectory. REG: target region, UR10: robot manipulator end effector trajectory, REF: reference trajectory.

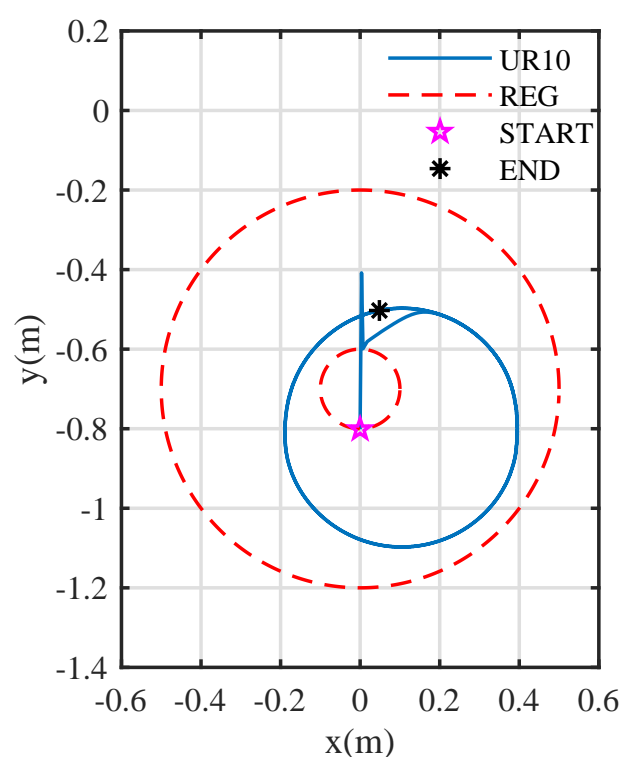

Fig. 10. Experimental case I: 2D view of the end effector trajectory. REG: target region, UR10: robot manipulator end effector trajectory, START: starting point, END: ending point.

s. The 3D view of the robot manipulator trajectory is shown in Fig. 5 along with the potential energy function shown in Fig. 6. The robot manipulator remains stable in the presence of the model uncertainty and external disturbance. After the disturbance is applied, the manipulator can remain in the target region and keep tracking the moving target region. The joints

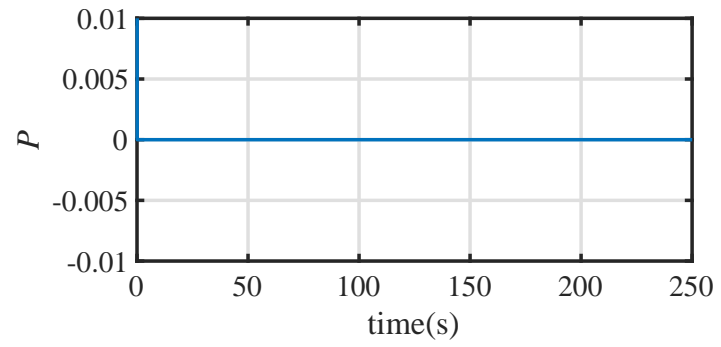

Fig. 11. Experimental case I: potential energy function.

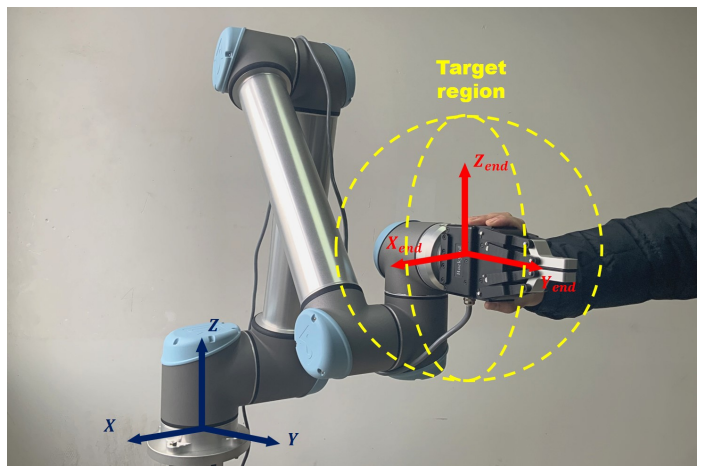

Fig. 12. Experimental case II: human robot interaction illustration. .

angles and control inputs are shown in Fig. 7. The simulation results have successfully validated the robustness of the UDEbased robust region tracking control algorithm for rejecting 


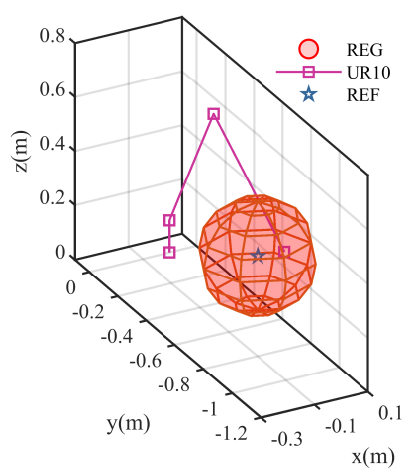

$t=0 \mathrm{~s}$

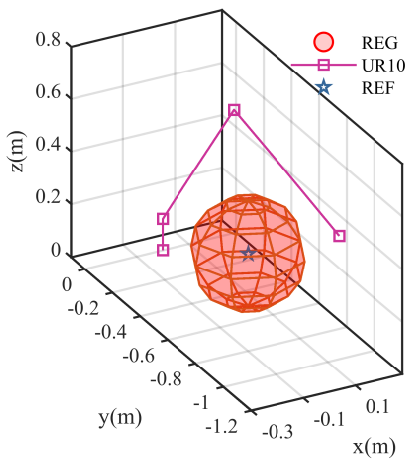

$t=7.6 \mathrm{~s}$

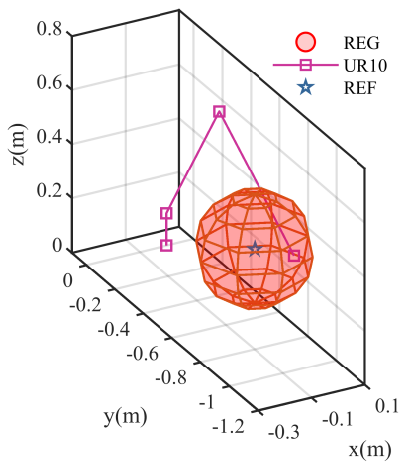

$t=13 \mathrm{~s}$

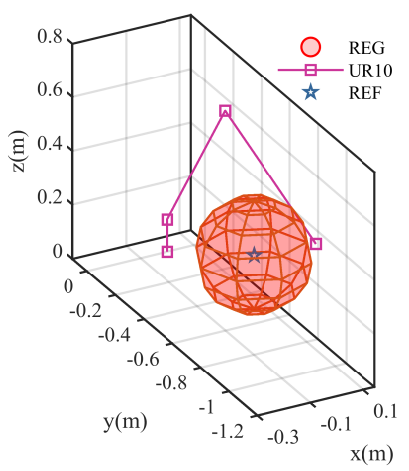

$t=4 \mathrm{~s}$

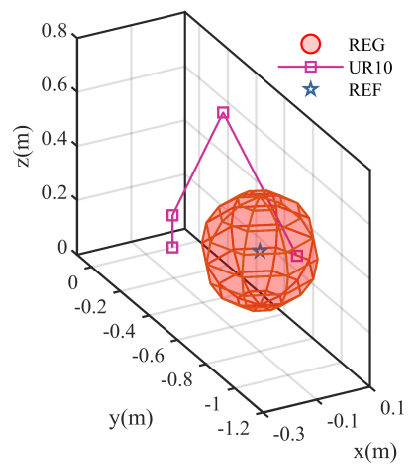

$t=10 \mathrm{~s}$

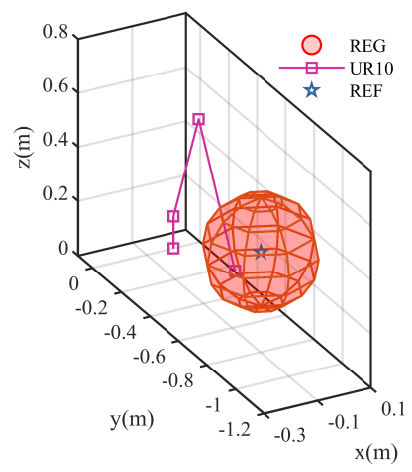

$t=14 \mathrm{~s}$

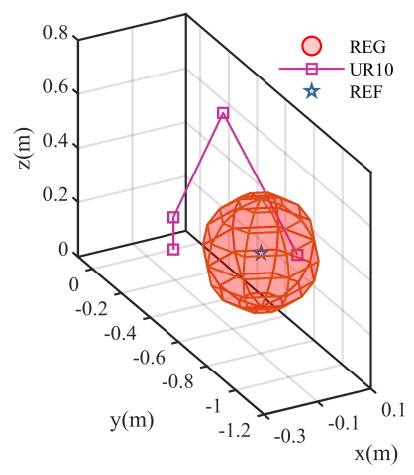

$t=7 \mathrm{~s}$

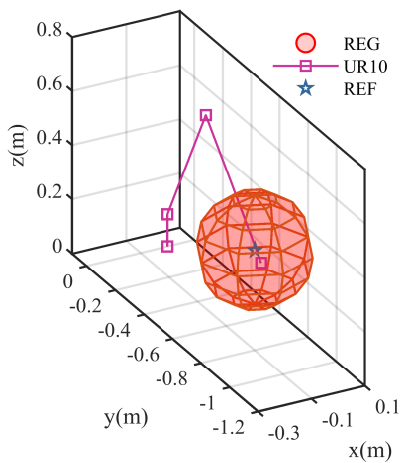

$t=10.4 \mathrm{~s}$

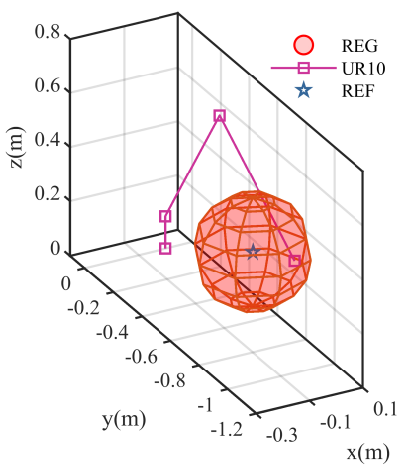

$t=19 \mathrm{~s}$

Fig. 13. Experimental case II: human-robot interaction: 3D view of the end effector trajectory. REG: target region, UR10: robot manipulator end effector trajectory, REF: reference trajectory.

the model uncertainty and external disturbance.

\section{B. Experimental Results}

To ensure the propose algorithm can be implemented on real robot manipulator platform, the experiment is conducted with the setup shown in Fig. 8. The host computer has a Core-i7 3.00 Ghz octa-core processor and a 32 GB memory. The operating system is Linux Ubuntu 18.04 and the Robot Operating System (ROS) Melodic is installed as the software framework for robot state data acquisition, controller implementation and communication. The joint angles and joint angular velocities of the UR 10 robot are sent to host computer, where the controller is implemented. Then host computer sends the control commands to the manipulator control box CB 3 through TCP/IP protocol at the update rate of $125 \mathrm{~Hz}$.
1) Experimental Case I: Moving Region Trajectory Tracking: The objective of the experiment is to let the end-effector of the UR10 robot to track the moving target region. The radius of the region and reference trajectory are $r=0.2 \mathrm{~m}$ and $\mathbf{x}_{o}=[0.3 * \cos (0.05 * t),-0.8+0.3 * \sin (0.05 * t), 0.2]^{\top}$, respectively. The initial positions of the lower three joints set as $\left[\begin{array}{lll}\pi / 2 & -\pi / 3 & \pi / 2\end{array}\right]^{\top}$ and the upper three joints are kept as fixed value of $\left[\begin{array}{lll}3 \pi / 4 & -\pi / 2 & 0\end{array}\right]^{\top} \mathrm{rad}$. The end effector trajectory are shown in 3D and 2D views in Fig. 9 and Fig. 10 , respectively. The time evolution of the potential energy function is shown in Fig. 11. This experimental results validate the effectiveness of the proposed approach for implementation on real robot manipulator platform to accomplish the moving trajectory tracking task. 


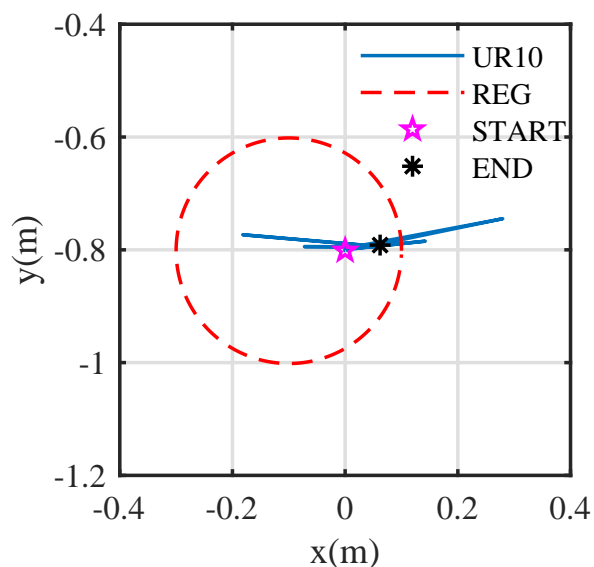

Fig. 14. Experimental case II: 2D view of the end effector trajectory. REG: target region, UR10: robot manipulator end effector trajectory, START: starting point, END: ending point.

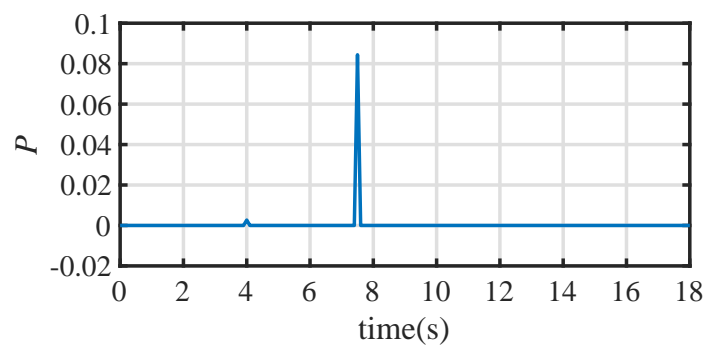

Fig. 15. Experimental case II: potential energy function.

2) Experimental Case II: Human-Robot Interaction: The control objective is to make the robot manipulator comply with the human operator within the target region. The initial positions of the lower three joints are set as the same as the experimental case I. The experimental procedures are as follows. Firstly, the robot manipulator is controlled to be stabilized at the target region with the center of $\mathrm{x}_{o}=$ $[-0.10,-0.80,0.37]^{\top}$ and the radius of $r=0.2 \mathrm{~m}$. After the robot manipulator is stabilized within the target region, the operator randomly drag the robot manipulator around to test the compliance, as shown in Fig. 12. The two and three dimensional views of the end effector trajectory are shown in Fig. 13 and Fig. 14, respectively. The potential energy function is shown in Fig. 15. It can be seen that when the operator drag the robot manipulator end effector within the target region, it exhibits compliant behavior and stays at the point where the manipulator release the end effector. When the robot manipulator end effector is outside the target region, the control algorithm can bring the end effector back to the target region. Based on the experimental results, it can be concluded that the proposed controller can ensure the complaint human robot interaction within the target region.

\section{CONClusion}

To deal with the practical challenges associated with the deployment of the robot manipulator for nursing tasks, includ- ing compliant human robot interaction, nonlinearity, model uncertainty and external disturbance, the UDE-based robust region tracking controllers were developed. The UDE was integrated into the region reaching control framework with the backstepping technique. The proposed approach ensured the robot manipulator track the moving target trajectory with sufficient robustness in the presence of model uncertainty and external disturbance. The compliance property for human robot interaction has also been demonstrated by dragging the robot manipulator within the target region. The effectiveness and performance of the developed control algorithm has been validated through both simulation and experimental studies with a UR 10 robot manipulator.

\section{ACKNOWLEGMENT}

The work has been financially supported by National Natural Science Foundation of China (Grant No. 51805449).

\section{REFERENCES}

[1] G.-Z. Yang, B. J. Nelson, R. R. Murphy, H. Choset, H. Christensen, S. H. Collins, P. Dario, K. Goldberg, K. Ikuta, N. Jacobstein, D. Kragic, R. H. Taylor, and M. McNutt, "Combating COVID-19-the role of robotics in managing public health and infectious diseases," Science Robotics, vol. 5, no. 40, May 2020.

[2] Y. Shen, D. Guo, F. Long, L. A. Mateos, H. Ding, Z. Xiu, R. B. Hellman, A. King, S. Chen, C. Zhang, and H. Tan, "Robots under COVID-19 Pandemic: A comprehensive survey," IEEE Access, vol. 9, pp. 1590-1615, Dec. 2021.

[3] WHO coronavirus disease (COVID-19) dashboard. (Accessed 2021, January 27). [Online]. Available: https://covid19.who.int/

[4] The Lancet, "COVID-19: protecting health-care workers," The Lancet, vol. 395, no. 10228, p. 922, Mar. 2020.

[5] Z. Li, P. Moran, Q. Dong, R. J. Shaw, and K. Hauser, "Development of a tele-nursing mobile manipulator for remote care-giving in quarantine areas," in Proceedings of IEEE International Conference of Robotics and Automation, 2017, pp. 3581-3586.

[6] G. Yang, H. Lv, Z. Zhang, L. Yang, J. Deng, S. You, J. Du, and H. Yang, "Keep healthcare workers safe: Application of teleoperated robot in isolation ward for COVID-19 prevention and control," Chinese Journal of Mechanical Engineering, vol. 33, no. 1, p. 47, Jun. 2020.

[7] L. Robotics. Lifeline robotics swab robot. (Accessed 2021, January 27). [Online]. Available: https://www.lifelinerobotics.com/\#careebo-1lr-s1

[8] S. Wang, K. Wang, R. Tang, J. Qiao, H. Liu, and Z.-G. Hou, "Design of a low-cost miniature robot to assist the COVID-19 Nasopharyngeal swab sampling," IEEE Transactions on Medical Robotics and Bionics, Nov. 2020, DOI:10.1109/TMRB.2020.3036461.

[9] S. Wu, K. Li, R. Ye, Y. Lu, J. Xu, L. Xiong, A. Cui, Y. Li, C. Peng, and F. Lv, "Robot-assisted teleultrasound assessment of cardiopulmonary function on a patient with confirmed COVID-19 in a cabin hospital," Advanced Ultrasound in Diagnosis and Therapy, vol. 4, no. 2, p. 128 Jun. 2020.

[10] W. E. Dixon, "Adaptive regulation of amplitude limited robot manipulators with uncertain kinematics and dynamics," IEEE Transactions on Automatic Control, vol. 52, no. 3, pp. 488-493, Mar. 2007.

[11] D. Chen, S. Li, W. Li, and Q. Wu, "A multi-level simultaneous minimization scheme applied to jerk-bounded redundant robot manipulators," IEEE Transactions on Automation Science and Engineering, vol. 17, no. 1, pp. 463-474, Jan. 2020.

[12] Y. Huang, Y. S. Yong, R. Chiba, T. Arai, T. Ueyama, and J. Ota, "Kinematic control with singularity avoidance for teaching-playback robot manipulator system," IEEE Transactions on Automation Science and Engineering, vol. 13, no. 2, pp. 729-742, Apr. 2016.

[13] M. Jin, S. H. Kang, P. H. Chang, and J. Lee, "Robust control of robot manipulators using inclusive and enhanced time delay control," IEEE/ASME Transactions on Mechatronics, vol. 22, no. 5, pp. 21412152, Jun. 2017.

[14] H. Wang, "Adaptive control of robot manipulators with uncertain kinematics and dynamics," IEEE Transactions on Automatic Control, vol. 62 , no. 2, pp. 948-954, Feb 2017. 
[15] C. C. Cheah, S. Kawamura, and S. Arimoto, "Feedback control for robotic manipulator with an uncertain jacobian matrix," Journal of Robotic Systems, vol. 16, no. 2, pp. 119-134, Feb. 1999.

[16] C. C. Cheah, C. Liu, and J. J. E. Slotine, "Adaptive tracking control for robots with unknown kinematic and dynamic properties," The International Journal of Robotics Research, vol. 25, no. 3, pp. 283296, Mar. 2006.

[17] C. C. Cheah and H. C. Liaw, "Inverse jacobian regulator with gravity compensation: stability and experiment," IEEE Transactions on Robotics, vol. 21, no. 4, pp. 741-747, Aug. 2005.

[18] H. Wang, W. Ren, C. C. Cheah, Y. Xie, and S. Lyu, "Dynamic modularity approach to adaptive control of robotic systems with closed architecture," IEEE Transactions on Automatic Control, vol. 65, no. 6, pp. 2760-2767, Jun. 2020.

[19] M. A. Arteaga-Perez, J. Pliego-Jimenez, and J. G. Romero, "Experimental results on the robust and adaptive control of robot manipulators without velocity measurements," IEEE Transactions on Control Systems Technology, vol. 28, no. 6, pp. 2770-2773, Oct. 2020.

[20] J. Nubert, J. Kohler, V. Berenz, F. Allgower, and S. Trimpe, "Safe and fast tracking on a robot manipulator: Robust MPC and neural network control," IEEE Robotics and Automation Letters, vol. 5, no. 2, pp. 30503057, Apr. 2020.

[21] A. Ferrara and G. P. Incremona, "Design of an integral suboptimal second-order sliding mode controller for the robust motion control of robot manipulators," IEEE Transactions on Control Systems Technology, vol. 23, no. 6, pp. 2316-2325, Nov. 2015.

[22] J. Lee, P. H. Chang, and M. Jin, "An adaptive gain dynamics for time delay control improves accuracy and robustness to significant payload changes for robots," IEEE Transactions on Industrial Electronics, vol. 67, no. 4, pp. 3076-3085, Apr. 2020.

[23] Q.-C. Zhong and D. Rees, "Control of uncertain LTI systems based on an uncertainty and disturbance estimator," ASME Journal of Dynamic Systems, Measurement, and Control, vol. 126, no. 4, pp. 905-910, Mar. 2004.

[24] Q. Lu, B. Ren, and S. Parameswaran, "Uncertainty and disturbance estimator-based global trajectory tracking control for a quadrotor," IEEE/ASME Transactions on Mechatronics, vol. 25, no. 3, pp. 15191530, Jun. 2020.

[25] B. Ren, J. Dai, and Q. Zhong, "UDE-based robust output feedback control with applications to a piezoelectric stage," IEEE Transactions on Industrial Electronics, vol. 67, no. 9, pp. 7819-7828, Sep. 2020.

[26] S. Islam and X. P. Liu, "Robust sliding mode control for robot manipu- lators," IEEE Transactions on Industrial Electronics, vol. 58, no. 6, pp. 2444-2453, Jun. 2011.

[27] J. Choi, J. Baek, W. Lee, Y. S. Lee, and S. Han, "Adaptive model-free control with nonsingular terminal sliding-mode for application to robot manipulators," IEEE Access, vol. 8, pp. 169897-169907, Sep. 2020.

[28] Y. Wang, D. Wang, and S. Zhu, "Formation tracking of multi-vehicle systems in unknown environments using a multi-region control scheme," International Journal of Control, vol. 90, no. 12, pp. 2760-2771, Dec. 2017.

[29] J. W. Yu, X. H. Zhang, J. C. Ji, J. Y. Tian, and J. Zhou, "Region-Reaching Control of a Flexible-Joint Manipulator," Journal of Dynamic Systems, Measurement, and Control, vol. 142, no. 11, Jul. 2020, 114503.

[30] C.-H. Lin, Y.-Y. Su, Y.-H. Lai, and C.-C. Lan, "A spatial-motion assistas-needed controller for the passive, active, and resistive robot-aided rehabilitation of the wrist," IEEE Access, vol. 8, pp. 133951-133960, Jul. 2020.

[31] K. P. Michmizos, S. Rossi, E. Castelli, P. Cappa, and H. I. Krebs, "Robot-aided neurorehabilitation: A pediatric robot for ankle rehabilitation," IEEE Transactions on Neural Systems and Rehabilitation Engineering, vol. 23, no. 6, pp. 1056-1067, Mar. 2015.

[32] C. C. Cheah, D. Q. Wang, and Y. C. Sun, "Region-reaching control of robots," IEEE Transactions on Robotics, vol. 23, no. 6, pp. 1260-1264, Dec. 2007.

[33] S. P. Hou and C. C. Cheah, "Region tracking control for robot manipulators," in Proceedings of IEEE International Conference on Control Applications, 2007, pp. 1438-1443.

[34] X. Li and C. C. Cheah, "Adaptive regional feedback control of robotic manipulator with uncertain kinematics and depth information," in Proceedings of American Control Conference, 2012, pp. 5472-5477.

[35] X. Li, G. Chi, S. Vidas, and C. C. Cheah, "Human-guided robotic comanipulation: Two illustrative scenarios," IEEE Transactions on Control Systems Technology, vol. 24, no. 5, pp. 1751-1763, Sep. 2016.

[36] C. C. Cheah, X. Li, X. Yan, and D. Sun, "Simple pd control scheme for robotic manipulation of biological cell," IEEE Transactions on Automatic Control, vol. 60, no. 5, pp. 1427-1432, 2015.

[37] W. He, C. Xue, X. Yu, Z. Li, and C. Yang, "Admittance-based controller design for physical human-robot interaction in the constrained task space," IEEE Transactions on Automation Science and Engineering, vol. 17 , no. 4, pp. 1937-1949, Oct. 2020.

[38] B. Ren, S. S. Ge, T. H. Lee, and M. Krstic, "Region tracking control for multi-agent systems with high-order dynamics," in Proceedings of American Control Conference, 2013, pp. 1266-1271. 